FMF patients without Amyloidosis diagnosis (FMF(+) A (-)), and healthy controls (HC). The mean ages, TREM-1, C - reactive protein (CRP), and Creatinine levels of each group are shown in Table 1. TREM-1 levels were found to be significantly higher in $\mathrm{A}(+) \mathrm{FMF}(+)$ group than $\mathrm{FMF}(+) \mathrm{A}(-)$, and healthy control groups $(p=0.001$ and 0.002$)$. Nevertheless, this difference was not found in between $A(+)$ FMF $(+)$ and FMF(-) $A(+)(p=0.447)$. In addition, the TREM-1 levels of $F M F(+) A$ $(-)$, and healthy control groups were not different (0.532). In $A(+) F M F(+)$ group, 36 patients used colchicine with the mean dose of $1.9 \pm 0.8 \mathrm{mg} /$ day, 14 patients used anakinra, and 9 patients used canakinumab. In $F M F(+) A(-)$ group all 20 patients used colchicine with the mean dose of $2.8 \pm 0.9 \mathrm{mg} /$ day, 1 patient used anakinra, and 2 patients used canakinumab.

Table 1. Clinical Features of Patients and TREM-1 levels

\begin{tabular}{lcccc}
\hline & $\begin{array}{c}\mathbf{A}(+) \\
(\mathbf{n}=\mathbf{4 2})\end{array}$ & $\begin{array}{c}\mathbf{F M F}(-) \mathbf{A}(+) \\
(\mathbf{n}=\mathbf{5})\end{array}$ & $\begin{array}{c}\mathbf{F M F}(+) \mathbf{A}(-) \\
(\mathbf{n}=\mathbf{2 0})\end{array}$ & $\begin{array}{c}\mathbf{H C} \\
(\mathbf{n}=\mathbf{2 0})\end{array}$ \\
\hline Age & $43.9 \pm 12.9$ & $54.8 \pm 19$ & $35.3 \pm 9.64$ & $35.4 \pm 6.57$ \\
TREM-1 & $735.3 \pm 566.5$ & $1247.1 \pm 1349.2$ & $414.3 \pm 142.3$ & $439.2 \pm 104.6$ \\
CRP & $11.1 \pm 14.2$ & $51.3 \pm 98.3$ & $25.8 \pm 54$ & $1.8 \pm 1.7$ \\
Creatinine & $1.6 \pm 1.8$ & $3.28 \pm 4.17$ & $0.7 \pm 0.15$ & $0.7 \pm 0.15$ \\
\hline
\end{tabular}

Conclusion: In conclusion, TREM-1 is a proinflammatory marker found significantly high in AA-amyloidosis patients regardless of their FMF diagnosis. TREM-1 may be useful in AA-amyloidosis follow-up and early diagnosis since currently there is a deficit of an early diagnostic marker of amyloidosis. This study is a cross-sectional one so it is hard to reach a conclusion on the effectiveness of TREM-1 during regular FMF follow-up for the secondary prevention of amyloidosis. However, the sensitivity of TREM-1 as a marker cannot be denied in amyloidosis.

Disclosure of Interests: None declared

DOI: 10.1136/annrheumdis-2020-eular.3426

\section{AB0056 NATURAL ANTIBODIES AGAINST PHOSPHORYLCHOLINE AND MALONDIALDEHYDE DURING THE FIRST TWO YEARS OF LIFE: IMPLICATIONS FOR RHEUMATIC DISEASE}

D. Thiagarajan ${ }^{1}$, S. Lundström¹, G. Pershagen ${ }^{1}$, C. Almqvist Malmros ${ }^{1}$, E. Andolf ${ }^{1}$, A. Hedman ${ }^{1}$, O. Berg ${ }^{1}$, N. Oparina ${ }^{1}$, J. Frostegård ${ }^{1} .{ }^{1}$ Karolinska Institutet, Stockholm, Sweden

Background: Antibodies against phosphorylcholine (anti-PC) have potentially protective properties in both atherosclerosis and rheumatic disease. IgM anti-PC could play a role in SLE being associated with protection, also in relation to atherosclerotic plaques and vulnerable plaques in $\mathrm{SLE}^{1}$ and being a non-responder to biologics in RA. ${ }^{1}$ We reported potential mechanisms by which anti-PC could be protective: 1:anti-inflammatory; 2: inhibits uptake of oxLDL in macrophages, 3: inhibits cell death. ${ }^{1} 4$ : anti-PC (and anti-MDA) increases clearance of human dead cells which could be of importance not especially in SLE; ${ }^{2}$ : anti-PC increases $T$ regulatory cells in SLE-patients' $T$ cells from a low level and also in atherosclerosis, with implications for both conditions. ${ }^{3}$ Also antibodies against malondialdehyde (anti-MDA) have interesting properties

Objectives: It is not known how these antibodies develop early in life and what may cause low levels. The objective is to determine this.

Methods: Antibodies were studied by ELISA in healthy pregnant women ( $n=105$; Born into life study) and their newborn children. Women were recruited before conception. Informed consent, questionnaires from parents and plasma sample was collected from children at birth from cord blood, at 1-year and 2 years after birth. Extracted antibodies were compared using a proteomics de novo sequencing approach.

Results: Children were born with very low levels of IgM anti-PC, while IgM antiMDA was present at birth,. Both IgM anti-PC and anti-MDA increased during the first two years of life, but IgM anti-PC in contrast to IgM anti-MDA was still significantly lower than mothers'. IgG anti-PC decreased after 1 year, but reached similar levels as mothers' after 2 years while IgG anti-MDA reached similar levels as mothers' already after one year. Proteomics peptide sequencing analysis indicates large peptide sequence variation without specific clone expression during early stage of life compared to the adult stage for which specific peptide sequences dominated. Conclusion: IgM anti-PC levels develop much slower than anti-MDA and are still relatively low at 2 years. We hypothesize that anti-PC is developed by a combination of pre-programming and exposure to the external world, where infectious agents may play a role. For anti-MDA pre-programming is likely to play a major role and at an earlier stage than for anti-PC.

References:

[1] Frostegard J. Immunity, atherosclerosis and cardiovascular disease. BMC Med. 2013;11:117.

[2] Rahman M, Sing S, Golabkesh Z, Fiskesund R, Gustafsson T, Jogestrand T, Frostegard AG, Hafstrom I, Liu A and Frostegard J. IgM antibodies against malondialdehyde and phosphorylcholine are together strong protection markers for atherosclerosis in systemic lupus erythematosus: Regulation and underlying mechanisms. Clin Immunol. 2016;166-167:27-37.

[3] Sun J, Lundstrom SL, Zhang B, Zubarev RA, Steuer J, Gillgren P, Rahman M, Ajeganova S, Liu A and Frostegard J. IgM antibodies against phosphorylcholine promote polarization of $\mathrm{T}$ regulatory cells from patients with atherosclerotic plaques, systemic lupus erythematosus and healthy donors. Atherosclerosis. 2018;268:36-48.

Disclosure of Interests: Divya Thiagarajan: None declared, Susanna Lundström: None declared, Göran Pershagen: None declared, Catharina Almqvis Malmros: None declared, Ellika Andolf: None declared, Anna Hedman: None declared, Oscar Berg: None declared, Nina Oparina: None declared, Johan Frostegård Grant/research support from: Unconditional competitive grant from Amgen, related only to PCSK9, not the topic of this abstract DOI: 10.1136/annrheumdis-2020-eular.5311

\section{$\mathrm{AB} 0057$ \\ THE ROLE OF NON-INFLAMMATORY MACROPHAGES INITIATING AND RESOLVING THE INFLAMMATORY RESPONSE OF MONOSODIUM URATE IN MICE}

Y. J. Huang ${ }^{1}$, L. C. Wang ${ }^{1}$, C. F. Kuo ${ }^{1} .^{1}$ Division of Rheumatology, Allergy and Immunology, Chang Gung Memorial Hospital and Chang Gung University, Taoyuan City, Taiwan, Republic of China

Background: Gout is the most common chronic inflammatory arthritis around the world which is associated with many conditions that affect longevity and well-being, such as metabolic syndrome, cardiovascular diseases, and renal diseases. [1]

Objectives: Gout is the most common inflammatory arthritis around the world Innate immunity has been implicated in gout inflammation in recent years. However, the phenomena of maintenance of 'asymptomatic' hyperuricemia, the spontaneous resolution of acute gouty arthritis and non-inflammatory tophus and its association with immune regulation are still elusive. We propose that residen macrophages may be a key player in the suppression of gouty inflammation. To address this hypothesis, we used non-inflammatory macrophages to explore the role played in initiating or resolving the inflammatory response.

Methods: Bone marrow-derived macrophages (BMDM) were stimulated with monosodium rate (MSU), then analyzed the expression of RNA and protein of inflammatory cytokines, including IL-1 $\beta$, IL-18 and TNF-a. In addition, we also observe the ability of macrophage to phagocyte and hydrolyze MSU crystal. Results: Our results indicate that MSU alone could not induce IL-1 $\beta, I L-18$, and TNF-a mRNA expression and protein production. However, when macrophages were pre-stimulated with lipopolysaccharide (LPS) or MSU, as well as in combination the production of IL-1 $\beta$ and IL-18 were significantly increased. Further more, MSU maybe amplifies LPS-induced protein production of IL-1 $\beta$ and IL-18 but not in TNF-a. A temporal delay in the correlation between mRNA expression and protein production was shown. The results also indicated that macrophages could not only phagocytize MSU crystals but may also hydrolyze MSU crystals. Conclusion: These data indicate that MSU crystal alone is insufficient to induce pro-inflammatory cytokines production, however, when exposed to an infectious source of infection, it will amplify the inflammatory response and there is a synergistic effect between MSU and LPS. This may explain the diverse clinic phenomena of 'asymptomatic' hyperuricemia, non-inflammatory tophus and acute gouty arthritis. The high efficiency of phagocytosis and hydrolyzed MSU crystals of macrophages may explain the spontaneous regression of acute gouty arthritis. These findings may provide a new therapy for the prevention and treatment of acute gout attacks.

References:

[1] Kuo CF, Grainge MJ, Mallen C, Zhang W, Doherty M. Comorbidities in patients with gout prior to and following diagnosis: case-control study. Annals of the rheumatic diseases. 2014 Nov 14.
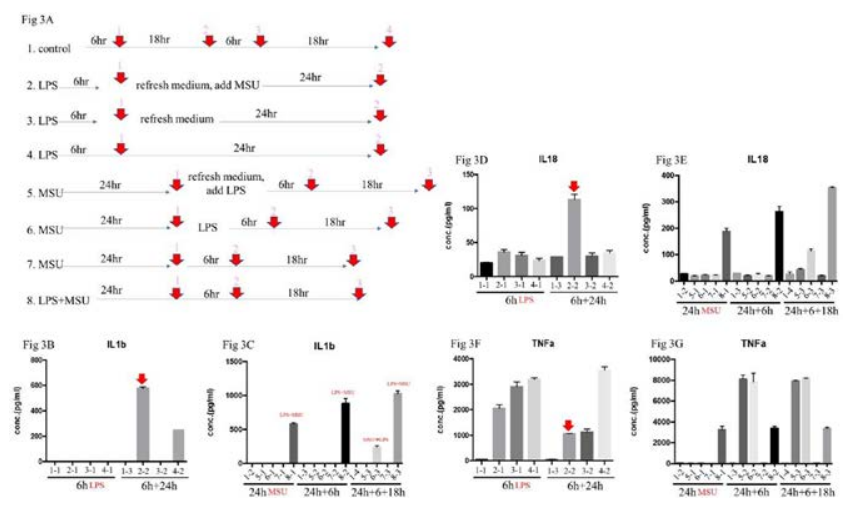


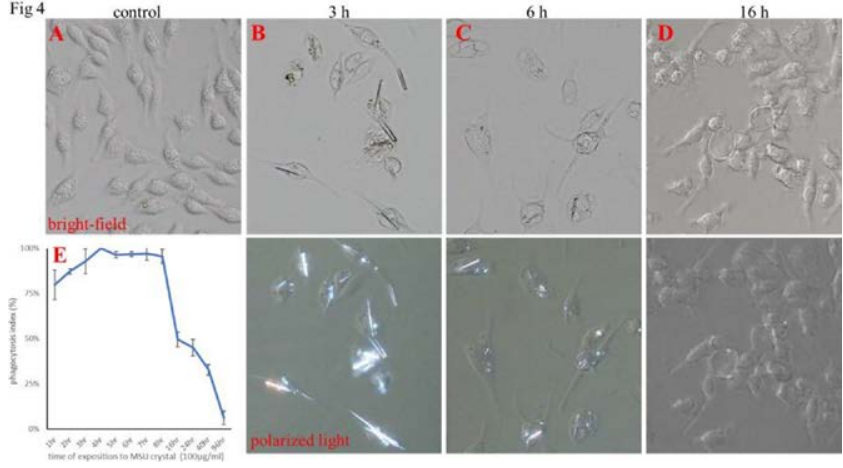

Disclosure of Interests: None declared DOI: 10.1136/annrheumdis-2020-eular.335

\section{$\mathrm{AB} 0058$ \\ CELL-TYPE SPECIFIC REGULATION OF IL-1R SIGNALING BY R835, A DUAL IRAK1/4 INHIBITOR}

S. Braselmann ${ }^{1}$, E. Tai ${ }^{1}$, R. Frances ${ }^{1}$, C. Young ${ }^{1}$, V. Markovtsov ${ }^{1}$, E. Masuda ${ }^{1}$, V. Taylor ${ }^{1}{ }^{1}$ Rigel Pharmaceuticals, South San Francisco, United States of America

Background: Interleukin-1 beta (IL-1b) is a key mediator of the inflammatory response and is known to exacerbate damage during chronic disease and acute tissue injury. Through association with the adaptor protein Myd88, interleukin receptor associated kinases (IRAK) 1 and 4 initiate signaling downstream of $\mathrm{IL}-1 \mathrm{Rs}$ resulting in the activation of the NFkB and MAPK pathways and the production of proinflammatory cytokines (1). IL-1Rs are broadly expressed across cell types and little is known about differences in signaling between cell types and the role of IRAK1 and IRAK4 kinase activity.

Objectives: We have identified a potent and selective IRAK1/4 inhibitor, R835, that substantially suppressed the elevation of LPS (TLR4 agonist)-induced serum cytokines in healthy human volunteers in a recent phase 1 study. The aim of this study was to evaluate the effect of R835 on IL-1R signaling in primary human fibroblasts and endothelial cells.

Methods: Human dermal fibroblasts, lung fibroblasts or endothelial cells were stimulated with IL-1b and the effect of R835 on the signaling pathway was evaluated by western blotting. Human dermal fibroblasts were stimulated with different amounts of IL-1b to evaluate both the signaling pathways activated and the cytokines produced. The ability of R835 to inhibit cytokine production induced by high or low amounts of IL-1b in dermal fibroblasts was assessed.

Results: In human endothelial cells, inhibition of IRAK1/4 kinases with R835 resulted in a block of IL-1b-induced IRAK4 phosphorylation, IRAK1 degradation and downstream NFkB, p38 and JNK activation. In contrast, in both human primary dermal and lung fibroblasts stimulated with IL-1b, we observed potent inhibition of IRAK4 phosphorylation, IRAK1 degradation, and downstream JNK phosphorylation, but no inhibition of NFkB pathway proteins and only weak inhibition of p38. Upon titration of IL-1b we observed that dermal fibroblasts produced $\mathrm{IL}-8$ and GRO in response to low levels of IL- $1 \mathrm{~b}(20 \mathrm{pg} / \mathrm{ml})$, and produced additional cytokines including G-CSF and GM-CSF with higher levels of IL-1b (400pg/ $\mathrm{ml})$. In the presence of low levels of IL-1b $(20 \mathrm{pg} / \mathrm{ml})$, we observed a weak activation of NFkB pathway proteins and p38, compared to a very robust NFkB, p38 and additional JNK activation in the presence of higher levels of IL-1b (400pg/ $\mathrm{ml}$ ). Consistent with these results, in dermal fibroblasts, R835 showed little to no inhibition of IL-8 and GRO induced by low levels of IL- $1 \mathrm{~b}$, but potently inhibited G-CSF and GM-CSF induced by high levels of IL-1b where JNK was activated. Conclusion: This study has elucidated signaling differences between cell types downstream of the IL-1R. In endothelial cells, as in myeloid cells, the kinase activity of IRAK1 and IRAK4 is required for the activation of all downstream signaling. Unexpectedly, in human fibroblasts, IRAK1/4 kinase activity appears to primarily regulate the JNK pathway, and not the NFkB pathway. Concomitant with that, only the cytokines induced by the additional activation of JNK in fibroblasts are regulated by a dual IRAK1/4 inhibitor. Clinically, an IRAK1/4 inhibitor may show select inhibition of IL-1b-induced cytokines depending on the tissue and cell type involved in inflammation.

References:

[1] Flannery S, Bowie A G. The interleukin-1 receptor-associated kinases: Critical regulators of innate immune signaling. Biochemical Pharmacology, Volume 80, Issue 12, 15 December 2010, Pages 1981-1991.

Disclosure of Interests: Sylvia Braselmann Shareholder of: Shareholder of Rigel Pharmaceuticals, Employee of: Employee of Rigel Pharmaceuticals, Ernest Tai Shareholder of: Rigel Pharmaceuticals, Employee of: Rigel Pharmaceuticals, Roy Frances Shareholder of: Rigel Pharmaceuticals, Employee of: Rigel Pharmaceuticals, Chi Young Shareholder of: Rigel Pharmaceuticals, Employee of:
Rigel Pharmaceuticals, Vadim Markovtsov Shareholder of: Rigel Pharmaceuticals, Employee of: Rigel Pharmaceuticals, Esteban Masuda Shareholder of: Rigel Pharmaceuticals, Employee of: Rigel Pharmaceuticals, Vanessa Taylor Shareholder of: Rigel Pharmaceuticals, Employee of: Rigel Pharmaceuticals DOI: 10.1136/annrheumdis-2020-eular.3852

\section{$\mathrm{AB} 0059$ \\ CLINICAL SIGNIFICANCE OF CIRCULATING MYELOID-DERIVED SUPPRESSOR CELLS IN PATIENTS WITH ANKYLOSING SPONDYLITIS}

A. Morenkova ${ }^{1}$, M. Tikhonova ${ }^{1}$, T. Tyrinova ${ }^{1}$, E. Batorov ${ }^{1}$, A. Sizikov ${ }^{2}$, O. Chumasova ${ }^{2}$, A. Sulutian ${ }^{2}$, V. Koksharova ${ }^{2}$, D. Orlov ${ }^{3}$, E. Chernykh ${ }^{1} .{ }^{1}$ Federal State Budgetary Scientific Institution Research Institute of Fundamental and Clinical Immunology, Laboratory of Cellular Immunotherapy, Novosibirsk, Russian Federation; ${ }^{2}$ Federal State Budgetary Scientific Institution Research Institute of Fundamental and Clinical Immunology, Rheumatology, Novosibirsk, Russian Federation; ${ }^{3}$ State Autonomous Healthcare Institution of the Novosibirsk Region City Center for Clinical Immunology, Novosibirsk, Russian Federation

Background: Myeloid-derived suppressor cells (MDSCs) represent heterogeneous population of immature myeloid cells with immunosuppressive functions. The important role of MDSCs is indicated for cancer, but their role in autoimmune pathology is currently controversial. Considering the clinical heterogeneity of ankylosing spondylitis (AS) and involvement of innate immunity in AS pathophysiology the investigation of the MDSC role in AS is of great interest.

Objectives: The aim of our study is to investigate the number of MDSC subsets in AS patients with different clinical manifestations, activity, disease duration, and treatment options and to evaluate the ability of MDSCs to mediate immunosuppressive function in AS patients.

Methods: The study included 34 patients with AS. Ankylosing Spondylitis Disease Activity Score (ASDAS) was used to assess disease activity and high activity was determined as $A S D A S \geq 2.1$. The frequencies of monocytic (M-MDSC) (HLADR-CD14 +), granulocytic (G-MDSC) (lin-HLADR-CD33+ CD66 +) and early-stage (eMDSC) (lin-HLADR-CD33 + CD66-) MDSCs and biomarkers of MDSCc functional activity including of Arg-1, IDO, PDL1 were determined in the peripheral blood by flow cytometry.

Results: We found significant elevation in the frequency of both M-MDSC and G-MDSC in the total group of patients compared to healthy controls $(\mathrm{HC})$ $(P=0.00006$ and $P=0.008$ respectively), while eMDSCs did not differ from $\mathrm{HC}$. Analysis of MDSCs populations in patient subgroups showed expansion of G-MDSCs in patients with axial plus peripheral damages $(P=0.004)$, while M-MDSCs were elevated regardless of the presence $(P=0.002)$ or absence $(P=0.001)$ of peripheral manifestations. Moreover, the percentage of M-MDSCs was positively correlated with ASDAS in patients with axial disease only $(R=0.8 ; P=0.03)$. Patients with low activity of disease demonstrated significant elevation of only M-MDSCs compared with $\mathrm{HC}(\mathrm{P}=0.001)$. Patients who had high activity of disease had increase in both M-MDSCs and G-MDSCs ( $\mathrm{P}=0.008$ and $\mathrm{P}=0.005$ respectively). By comparing the frequency of MDSCs in patient groups with different AS duration we showed increase in percentage of both M-MDSCs and G-MDSCs in patients with relatively short duration of disease ( $<M e=11.5$ years) $(P=0.002$ and $P=0.005$ respectively) and elevation in M-MDSCs only in patients with longer AS duration $(P=0.0003)$ Compared with patients receiving conventional therapy (NSAIDs, csDMARDs), patients who received biological agents (TNFa inhibitors) had lower disease activity but despite this showed elevated frequencies of M-MDSCs and PMN-MDSCs, comparable to patients receiving conventional therapy. Of note, M-MDSCs in AS patients had increased expression of $P D L-1$ and IDO $(P=0.04$ and $P=0.02$ respectively) and similar to $\mathrm{HC}$ expression of Arg-1. The expression of Arg-1, IDO, PDL1 in patients G-MDSCs did not differ from $\mathrm{HC}$.

Conclusion: The data obtained indicate that both M-MDSCs and G-MDSCs are elevated in AS patients. However, the increase of G-MDSCs is associated with peripheral manifestations of AS, high activity, longer duration, and the percentage of M-MDSCs was positively correlated with activity in patients with axial disease only. The unchanged expression of Arg-1, PDL-1 and IDO in G-MDSCs and enhanced expression of PDL-1 and IDO in M-MDSCs suggest MDSCs capacity to mediate immunosuppressive function in AS patients.

Disclosure of Interests: None declared

DOI: 10.1136/annrheumdis-2020-eular.2998

\section{AB0060 \\ STING AND PROINFLAMMATORY CYTOKINES IN SYNOVIAL FLUID OF PATIENTS WITH DIFFERENT ARTHRITIDES}

A. Scanu ${ }^{1}$, R. Luisetto ${ }^{2}$, F. Oliviero ${ }^{1}$, P. Galozzi ${ }^{1}$, A. Ortolan ${ }^{1}$, M. Lorenzin ${ }^{1}$, M. Felicetti ${ }^{1}$, A. Doria ${ }^{1}$, R. Ramonda ${ }^{1}$. 'University of Padova, Rheumatology Unit, Dept. of Medicine-DIMED, Padova, Italy; ${ }^{2}$ University of Padova, Dept. of Surgical Oncological and Gastroenterological Sciences, Padova, Italy 